conditions, pathogenic isolates of $F$. oxysporum produced rhizome infections, whereas other isolates did not. When $F$. oxysporum isolates were obtained by plating from various sources, the proportions which proved to be pathogenic were : 91 per cent from the rhizome or pseudostem of plants showing typical wilt ; 29 per cent from variously affected roots ; and 0 per cent from soil. The information now available suggests that the banana pathogen, like other parasitic forms of $F$. oxysporum, has a limited host range; however, it has now been shown to cause a limited root infection of Heliconia psittacorum and may possibly originate on species such as this related to the banana. An account is also given in the paper of the development of the disease in sites which had been replanted after previous infection and abandonment. New cases may arise both spontaneously and in association with pre-existing ones, the rate of appearance of each type tending to decline after an initial riso. Although, on the evidence, $F$. oxysporum f. cubense only survives for a relatively short period in infected plant material, owing to rapid decay, it nevertheless survives for many years in the soil. A marked increase in the population of the pathogen takes place a few months after wilt disease appears, but there is a decline after infected plants are removed. Wide variation was found in the ratio of non-pathogenic to pathogenic strains of $F$. oxysporum in soils, but it is usually very high. The possible mode of survival of the pathogen in soil is discussed.

\section{Forestry Abstracts}

Forestry is a science of region-wide husbandry and land use, and the training of the forester is based on many sciences; for example, he must survey and map both from the ground and the air, be able to construct, and certainly maintain, roads, bridges, cable-ways and buildings, and handle specialized machinery. These are in addition to his interest in his major object-the production of wood-which takes him a long way into applied biology, genetics, physics, mechanics and chemistry. In a recent number of Forestry Abstracts (16, No. 2; 1955 ; Commonwealth Forestry Bureau, Oxford) an article entitled "The Literature of Forestry : an Appraisal" gives the aims and objects of the Abstracts. "In view of its primary function--to act as an effective clearinghouse of information for scientists and research workers .... in forestry-ready access to all of the relevant literature is a constant basic objective of this and of every Bureau of the Commonwealth Agricultural Bureaux Organization. In the world of to-day, with scientific publishing an ill-ordered jungle, and procurement aggravated by post-war political barriers, such an object must remain impossible of attainment." In consequence, material of value to the forester's work must be watched for in a very wide range of non-forestry publications, from a microscopists' journal to the monograph in applied chemistry (of course, these difficulties are shared in varying measure by other sciences). A century ago, there was very little forestry literature published in the English language. The great expansion has taken place during the present century ; the most important part, but by no means the whole, is published in the bulletins, reports, etc., issued by research centres and in the leading scientific periodicals. In the article in Forestry Abstracts some interesting figures are given of the great increase in the mass of literature both in volume and variety of origin since the Second World War. In January 1947 periodicals numbered 379 and serial sets (including annual reports) 470 ; total 849 . In January 1955, periodicals were 555 and serial sets 755 ; total 1,310 . This mass of publications originated from nearly a hundred countries with more than thirty languages.

\section{Madrid Observatory Almanac for 1955}

A comparison of "The Nautical Almanac" with the "Anuario del Observatorio Astronómico de Madrid para 1955" (pp. $429+2$ plates. Madrid: Observatorio Astronómico, 1954 ; 40 pesetas) shows that, while practically the same ground is covered by each, the Spanish publication contains some additional matter which will prove useful to astronomers in other countries. In general, the "Anuario", which is published under the direction of Sr. Martin Lorón, follows the plan that has been in use for some years. Among the additional matter may be noticed a list of fortyfour periodic comets which have been observed from 467 B.C. up to A.D. 1942, the first of these being Halley's Comet and the last Comet Oterma. This is followed by a catalogue of the principal constellations, arranged in order of their right ascensions, then by another catalogue of clusters and nebulæ visible from Spain, and also one of double stars. The geographical positions of the chief observatories are given, after which there are 113 pages dealing with visual double stars. This section commences with a short historical sketch, starting with Riccioli's description in 1650 of $\zeta$ Urs. Maj., and including an account of the work done by various observers in this branch of astronomy up to recent times. Methods of observation, measurements by means of the interferometer, Hargreaves's comparison micrometer with diagrams explanatory of the principle, etc., are dealt with, and the section ends with a description, covering fifty pages, of the method of determination of binary orbits. This includes two tables for facilitating the computations, and everyone interested in the computation of the orbits of binaries will find this portion very helpful.

\section{Origin of Stellar Systems and Planets}

A LoNe article by Alexandre Dauvillier, dealing with the origin of galaxies, planetary systems, satellites, comets, etc. (l'origine de la terre, systèmes stellaires et planétaires : structure et origine), is to be found in La Revue Scientifique (No. 3324, JulyDecember 1953), the article being based on an annual inaugural address of the course of cosmic physics given at the Collège de France, Paris ; those interested in problems of cosmology will find much in it of considerable importance. The formation of planetary systems is, according to Dauvillier, merely a particular case of stellar interactions, resulting generally in the formation of double and multiple stars. It is estimated that our Galaxy must contain at the least two thousand million planetary systems, though many of the planets may have very eccentric orbits resembling those of comets; others may have planets more massive than Jupiter, and many other variations from what we find in our solar system are inevitable.

\section{Postgraduate Awards in British Commonwealth Universities}

THE Assoriation of Universities of the British Commonwealth has issued as a separate pamphlet Appendixes II and III of the Commonwealth Universities Yearbook, 1955 (pp. 117; from the Association at 36 Gordon Square, London, W.C.1). The first of these, which is printed in accordance 